\title{
Editorial
}

\section{Protection de la santé à la place de travail: une tâche complexe et multidisciplinaire}

\section{R. Bruppacher, D. Bauer}

L'étude des interactions entre les conditions de vie et la maladie forment une préoccupation principale de la médecine sociale et préventive. Les phénomènes à l'intérieur du corps sont corrélés aux influences de l'environnement social et physique (médecine sociale) afin de pouvoir trouver les moyens de protéger et de promouvoir la santé (médecine préventive).

Le travail et ses facteurs représentent pour les sciences de prévention un objet d'étude très important et extrêmement prometteur. Il est vrai que seule une petite partie de la vie se passe à la place de travail: 48 années dactivité professionnelle à raison de 44 heures par semaine correspondent à un peu plus de $100 \cdot 000$ heures. moins d'un sixième de la durée moyenne de la vie de 73 ans (640'000 heures). Mais cette période de travail est très importante car c'est la seule période de vie ou l'homme est exposé à autant de facteurs externes et artificiels sur lesquels il n'a que peu d'influence. L'étude de ces phénomènes amène à mieux connaítre la relation entre le domaine de travail et la santé, ce qui permettra ensuited daméliorer sensiblement les conditions de travail. Dans ce domaine, les intérêts des employeurs et des employés sont finalement très similaires sauf peut-être en ce qui concerne les buts à réaliser à court terme: sans postes de travail salubres il n'y aura pas d'industrie ou d'artisanat florissants.

Les circonstances particulières et la complexité de la place de travail moderne ont nécessité la création de disciplines spécialisées. aussi bien du côté clinico-thérapeutique de la médecine préventive que du côté technique: La médecine du travail étudie en premier lieu les modifications apparaissant dans le corps humain. dues aux conditions de travail. La physiologie du travail (ergonomie, "human factors engineering") par contre s'occupe des interactions entre l'être humain en bonne santé avec son environnement professionnel. Cette dernière spécialité trouve un complément très important pour le bien-être psychique au travail dans la psychologie du travail. La sécurité du travail. c'est à dire la prévention des accidents au sens classique, vise à analyser les causes d'accidents et cherche à les prévenir (la nouvelle LAA utilise le terme sécurité du travail aussi dans un sens général pour toutes les mesures de prévention de maladies et accidents professionnels). L'hygiène du travail enfin surveille et contrôle les nuisances a la place de travail. Cette discipline. plus encore que les précédentes. s'occupe de la protection et la promotion de la santé du travailleur et forme ainsi un thème approprié pour notre périodique.

Ayant publié dans le passé une multitude de contributions individuelles - surtout à la suite de séances de travail scientifiques - nous publions cette fois un numéro entièrement réservé à ce thème. La partie centrale est occupée par le rapport "Hygiène du Travail en Suisse" de la Commission correspondante de la société suisse de médecine, d'hygiène et de sécurité du travail. Afin de lui réserver une autonomie. ce rapport a une table des matières et une numérotation de pages individuelles*. On en déduit que l'nygiène du travail couvre un domaine vaste. chevauchant les disciplines mentionnées plus haut. La protection de la santé à la place de travail en effet est un devoir multidisciplinaire nécessitant la contribution de médecins orientés du côté clinique aussi bien qu'épidémiologique, des ingénieurs, physiciens, chimistes, économistes. psychologues, de même que des juristes et spécialistes d'assurance. Les autres contributions de ce cahier en fourniront la preuve. Ce numéro forme un complément au numéro $3 / 81$ "Médecine du travail en suisse". Espérons que ce numéro thématique puisse mieux faire connaître l'hygiène du travail et promouvoir la compréhension mutuelle ainsi que la collaboration des spécialistes. nécessaires pour remplir cette grande tấche commune.

Nous aimerions consacrer ce numéro à un pionnier de l'hygiène et de la physiologie du travail en Suisse. le professeur Etienne Grandjean, à l'occasion de son septantième anniversaire. Au cours de ses activités scientifiques multiples et extrèmement fructueuses à I'EPF zürich, il a su promouvoir comme personne les travaux interdisciplinaires se rattachant aux questions de la santé à la place du travail. Par sa pensée il a transmis à beaucoup de chercheurs son enthousiasme pour les sciences du travail et de la prévention. y compris les sousignés.
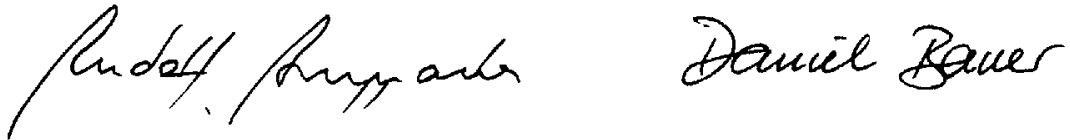

* Le rapport de la commission peut être obtenu séparément auprès du sécrétariat de la société suisse de médecine, d'hygiène et de sécurité du travail (c/o Caisse Nationale d'Assurance, 6002 Lucerne). 